\title{
The RNA sequence context defines the mechanistic routes by which yeast arginyl-tRNA synthetase charges tRNA
}

\author{
MARIE SISSLER, RICHARD GIEGÉ, and CATHERINE FLORENTZ \\ UPR 9002, IBMC du CNRS, 15 rue René Descartes, F-67084 Strasbourg Cedex, France
}

\begin{abstract}
Arginylation of tRNA transcripts by yeast arginyl-tRNA synthetase can be triggered by two alternate recognition sets in anticodon loops: C35 and U36 or G36 in tRNA ${ }^{\text {Arg }}$ and C36 and G37 in tRNA ${ }^{\text {Asp }}$ (Sissler M, Giegé R, Florentz C, 1996, EMBO J 15:5069-5076). Kinetic studies on tRNA variants were done to explore the mechanisms by which these sets are expressed. Although the synthetase interacts in a similar manner with tRNA ${ }^{A r g}$ and $\operatorname{tRNA} A^{A s p}$, the details of the interaction patterns are idiosyncratic, especially in anticodon loops (Sissler M, Eriani G, Martin F, Giegé R, Florentz C, 1997, Nucleic Acids Res 25:4899-4906). Exchange of individual recognition elements between arginine and aspartate tRNA frameworks strongly blocks arginylation of the mutated tRNAs, whereas full exchange of the recognition sets leads to efficient arginine acceptance of the transplanted tRNAs. Unpredictably, the similar catalytic efficiencies of native and transplanted tRNAs originate from different $k_{c a t}$ and $K_{m}$ combinations. A closer analysis reveals that efficient arginylation results from strong anticooperative effects between individual recognition elements. Nonrecognition nucleotides as well as the tRNA architecture are additional factors that tune efficiency. Altogether, arginyltRNA synthetase is able to utilize different context-dependent mechanistic routes to be activated. This confers biological advantages to the arginine aminoacylation system and sheds light on its evolutionary relationship with the aspartate system.
\end{abstract}

Keywords: aminoacylation identity; evolution; RNA-protein recognition; tRNA ${ }^{\text {Arg }}$; tRNA ${ }^{\text {Asp }}$

\section{INTRODUCTION}

Accuracy of translation relies on specific aminoacylation of tRNAs by their cognate aminoacyl-tRNA synthetases (aaRS). This specificity is governed by molecular signals within tRNAs, including positive elements responsible for specific recognition by cognate synthetases and negative elements hindering recognition by noncognate enzymes. Recognition sets have been established in a number of tRNAs (e.g., Giegé et al., 1993; McClain, 1993a, 1993b; Saks et al., 1994) and were shown to be constituted by a limited number of nucleotides and/or structural features. The concept of specificity calls for a unique combination of those elements for a given aminoacylation system and indeed many experimental data are in line with this view. However, for the arginine system, we have demonstrated that yeast arginyl-tRNA synthetase (ArgRS) has the unexpected property of recognizing indiscriminately two

Reprint requests to: Richard Giegé, UPR 9002, IBMC du CNRS, 15 rue René Descartes, F-67084 Strasbourg Cedex, France; e-mail: giege@ibmc.u-strasbg.fr. alternate sets of nucleotides within two host tRNAs (Sissler et al., 1996). Indeed, ArgRS aminoacylates its major tRNA ${ }^{\text {Arg }}$ isoacceptor thanks to the presence in its anticodon loop of C35 and, to a lesser extent, of U36 or G36. This set is designated as [C35U36] ${ }^{\mathrm{Arg}}$. Additionally, yeast ArgRS is also able to interact rather strongly with native tRNA ${ }^{\text {Asp }}$ and to mischarge this molecule with low efficiency (Ebel et al., 1973; Gangloff et al., 1973; Perret et al., 1990a). Arginylation becomes efficient with noncognate tRNA ${ }^{\text {Asp }}$ so far as it is deprived of modified nucleotides (Perret et al., 1990a; Pütz et al., 1994). For this unmodified substrate, arginylation is deeply related to the presence of residues C36 and G37, but is insensitive to the nature of $\mathrm{nt} 35$. This second and alternate recognition set is designated as [C36G37] ${ }^{\text {Asp }}$. Furthermore, contacts of yeast ArgRS on in vitro-transcribed tRNA ${ }^{\text {Arg }}$ (derived from the major isoacceptor) and tRNA ${ }^{\text {Asp }}$ have been established by footprinting with enzymatic and chemical probes (Sissler et al., 1997). They revealed that both transcripts interact with ArgRS along the D-arm side as typical for class I synthetases, and the anticodon loop, the region that contains the identity nucleotides. However, details 
of interaction patterns are idiosyncratic and indicate that recognition is governed by the synthetase.

The existence of two alternate identity sets that trigger catalysis by the same synthetase addresses new questions about the mechanisms leading to aminoacylation specificity. We recall that transplantation of individual arginine identity elements from one tRNA substrate into the other one has negative consequences on arginylation (Sissler et al., 1996) and suggests nonclassical behaviors of these elements. Further, the functional properties of a chimeric tRNA ${ }^{\mathrm{Arg}}$ with the anticodon loop of tRNA ${ }^{\text {Asp }}$ demonstrated that the framework in which the identity elements are embedded contributes to expression of arginine identity (Sissler et al., 1996).

This work investigates the mechanism of arginine identity expression and the precise interrelations between the two arginine identity sets within the tRNA $A^{\operatorname{Arg}}$ or tRNA ${ }^{\text {Asp }}$ frameworks. Functional analysis of a series of single and multiple tRNA $A^{\text {Arg }}$ and tRNA ${ }^{\text {Asp }}$ variants were aimed at defining the relationships between the individual identity elements in the two tRNA frameworks. Data indicate that arginylation of the in vitro transcripts is governed by alternate mechanisms according to the host tRNA. Not only does ArgRS interact differently with tRNA anticodon loops, but it also triggers efficient aminoacylation by alternate mechanisms as reflected by the kinetic analysis of the arginylation reactions. These findings open new evolutionary perspectives toward understanding tRNA aminoacylation identity.

\section{RESULTS AND DISCUSSION}

\section{Four efficient tRNA substrates of ArgRS behave differently kinetically}

Exchange of individual arginine identity nucleotides between tRNA ${ }^{\text {Arg }}$ and tRNA ${ }^{\text {Asp }}$ transcripts produces dramatic negative effects on their arginylation capacities (Sissler et al., 1996). To understand the duality of arginine identity expression, two chimera were constructed into which the complete recognition set of the other host molecule was transplanted. In these tRNAs, residual nucleotides from the native recognition set were removed by mutation. Sequences of variants, ${ }^{1}$ together with those of their wild-type counterparts, are displayed in Figure 1. Variant ArgU35C36G37, constituted by the tRNA $A^{\text {Arg }}$ framework, contains in its anticodon loop the [C36G37] ${ }^{\text {Asp }}$-recognition set. In this molecule, position 35 has been mutated to $U$ in order to remove

\footnotetext{
${ }^{1}$ Throughout the text, tRNA "body" means all nucleotides except those from the anticodon loop and "framework" means all nucleotides except the triplet 35,36 , and 37 containing the identity elements. Nomenclature of variants is as in Sissler et al. (1996), namely with bold characters for features occurring naturally in tRNA ${ }^{\mathrm{Arg}}$ and italics for nucleotides occurring naturally in tRNA ${ }^{A s p}$; wild-type tRNA transcripts are referred to as ArgWT and AspWT.
}

C35, the major identity nucleotide of the [C35U36] $]^{\mathrm{Arg}}$ recognition set. Inversely, variant AspC35U36A37, constituted by the tRNA ${ }^{\text {Asp }}$ framework, bears the [C35U36] ${ }^{\text {Arg }}$-recognition set and has G37 replaced by $A$ in order to remove the residual identity nucleotide of the [C36G37] ${ }^{\text {Asp }}$-recognition set.

The kinetic characteristics of the two chimera and the wild-type tRNAs are given in Table 1. The losses $(\mathbf{L})$ of arginylation efficiencies (or kinetic specificities) are expressed in comparison to wild-type in vitro-transcribed tRNA ${ }^{\text {Arg }}$, considered as the reference molecule throughout this work. All four tRNAs are efficient substrates of yeast ArgRS with charging efficiencies varying at most by a factor of 11 . Thus, transplantation of the complete recognition sets is well perceived by ArgRS, even if transplantations of single nucleotides dramatically impair arginylation of host tRNAs (e.g., $\mathbf{L}=2780$ after insertion of U36 into tRNA ${ }^{\text {Asp }}$, see Table 1). However, analysis of the individual kinetic parameters of the four tRNAs shows great variability.

A deeper insight comes from comparison of arginylation efficiencies within couples of variants. Comparing $\operatorname{Arg} U 35 C 36 G 37(\mathbf{L}=11)$ and AspWT $(\mathbf{L}=10)$ with ArgWT (grey arrows in Fig. 1) shows that the moderate loss in activity for the tRNA ${ }^{\text {Arg }}$ variant is due to strong compensations between affinity and catalytic rate $\left(1 / K_{m}\right.$ increases 10.5 -fold; $k_{c a t}$ decreases 115 fold), whereas with AspWT affinity remains constant and the 10 -fold loss is only due to a decreased $k_{\text {cat }}$. Similarly, comparing AspC35U36A37 with AspWT and Arg U35C36G37 (dashed arrows) reveals different levels of compensation brought by $K_{m}$ and $k_{c a t}$. Altogether, different combinations of relative $k_{c a t}$ and $K_{m}$ lead to similar losses of catalytic efficiencies.

Interestingly, ArgU35C36G37 with the [C36G37] ${ }^{\text {Asp _ }}$ recognition set acquires the efficiency of $\operatorname{AspWT}(\mathbf{L}=$ 11 versus 10), and AspC35U36A37 bearing the [C35U36] ${ }^{\mathrm{Arg}}$-recognition set is as active as ArgWT ( $\mathbf{L}=0.8$ versus 1$)$. In other words, the two couples of transcripts (linked by black arrows in Fig. 1), each sharing the same nucleotide triplet at positions 35,36 , and 37 (either C35U36A37 or U35C36G37), have a similar catalytic efficiency $(\mathbf{L} \sim 10$ or $\mathbf{L} \sim 1)$. This suggests that nucleotide combinations at positions 35-37 have a dominant role in arginylation whatever the tRNA framework.

Comparison of the kinetic data of the four arginine acceptors also informs about the role of the tRNA frameworks. First, ArgWT and AspC35U36A37, with the same arginine recognition set but not the framework, have a 4.7-fold difference in $K_{m}$ compensated by a 3.5 -fold difference in $k_{\text {cat }}$. Larger effects are observed for AspWT and Arg U35C36G37, where both $K_{m}$ and $k_{\text {cat }}$ vary by a factor of $\sim 11$.

The existence of such differences between individual kinetic parameters of transplanted and wild-type tRNAs is surprising because the anticodon loops of the four tRNAs possess all the elements needed to preserve a 


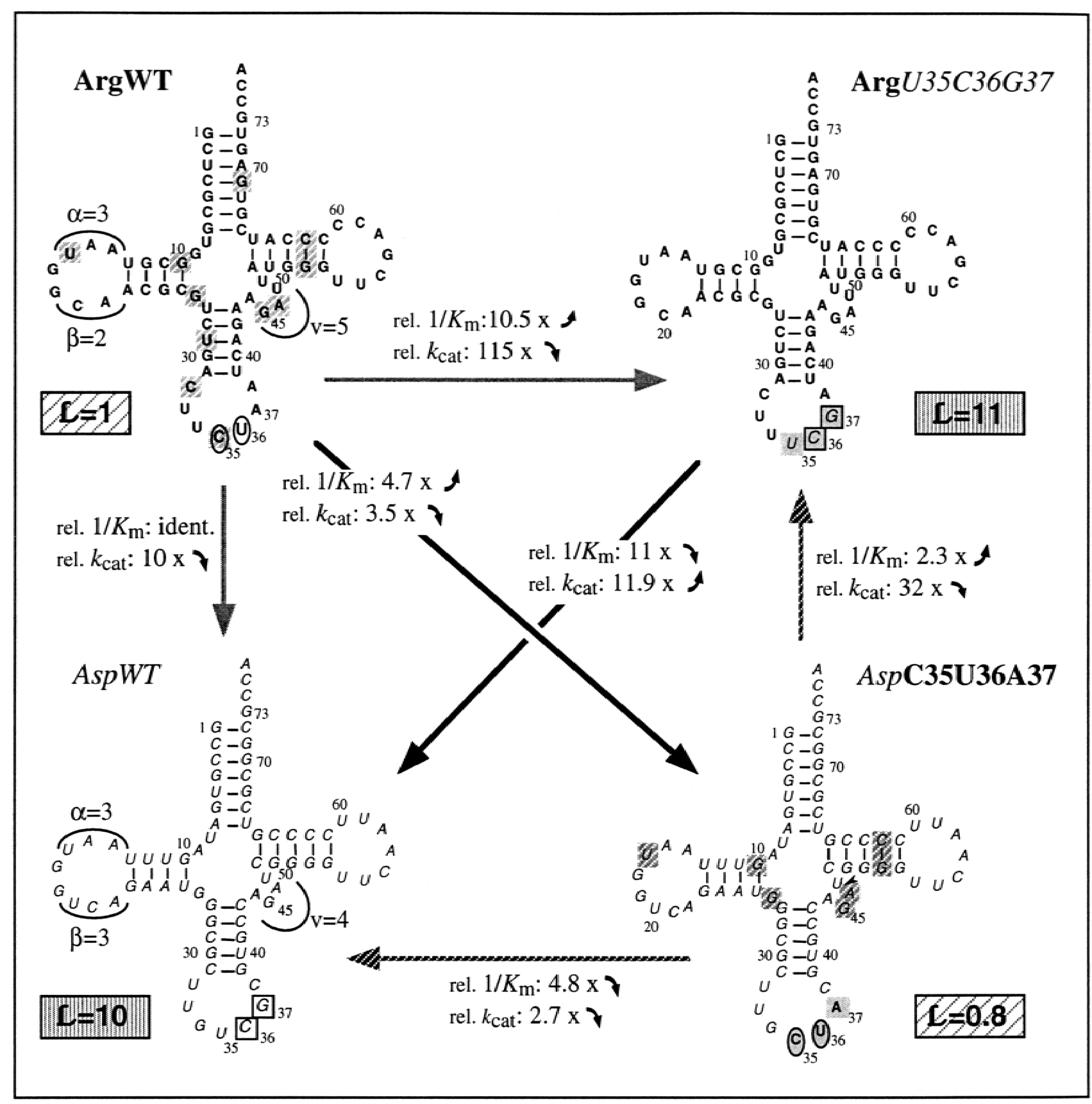

FIGURE 1. Relationship between four efficient tRNA substrates of yeast ArgRS. Wild-type in vitro transcripts of tRNA ${ }^{A r g}$ (ArgWT) and tRNA ${ }^{\text {Asp }}$ (AspWT) bearing each a different identity set within their anticodon loop (either circled or boxed) are the reference molecules. In both tRNAs, the original set has been removed and the alternate set introduced, allowing creation of variants ArgU35C36G37 and AspC35U36A37 (with mutated positions shaded). Bold characters correspond to nucleotides occurring naturally in tRNA Arg and italics for those occurring naturally in tRNA ${ }^{\mathrm{Asp}}$. $\mathbf{L}$ values are losses in aminoacylation efficiencies with ArgWT as reference. The functional relationships between tRNAs are given as $x$-fold increases or decreases of rel. $1 / K_{m}$ and rel. $k_{c a t}$-values ("rel." stands for "relative") and are symbolized by arrows pointing up or down, respectively. Nucleotides common to the five yeast tRNA ${ }^{A r g}$ isoacceptors (with the exception of conserved and semi-conserved residues) are shaded in the sequence of ArgWT; those common in tRNA ${ }^{\text {Arg }}$ isoacceptors and tRNA ${ }^{\text {Asp }}$ are shaded in the sequence of AspC35U36A37 (arrow indicates that a nucleotide is missing in the variable region of tRNA ${ }^{A s p}$ ). Sizes of $\alpha$ and $\beta$ domains and of variable region are indicated in ArgWT and AspWT (note that these sizes are not conserved in the arginine isoacceptors).

canonical anticodon loop structure (review by Dirheimer et al., 1995). Conserved U33 is maintained as well as semi-conserved pyrimidine 32 and purine 37 . However, residues 32 and 38 , which form a noncanonical base pair stacked over the anticodon stem, are different (C32-A38 or U32-C38) and, for some of the couples, the tRNA framework is different. We hypothesize that these differences account for the observed kinetic 
TABLE 1. Kinetic parameters for arginylation by yeast ArgRS of Arg/Asp chimera and of architectural tRNA variants derived from yeast tRNA ${ }^{\text {Arg }}$ and tRNA ${ }^{\text {Asp }}$.

\begin{tabular}{|c|c|c|c|c|c|c|}
\hline Transcripts & $\begin{array}{l}k_{\text {cat }} \\
\left(\mathrm{s}^{-1}\right)\end{array}$ & $\begin{array}{c}K_{m} \\
(\mathrm{nM})\end{array}$ & $\begin{array}{c}k_{\text {cat }} / K_{m} \\
\text { (relative) }\end{array}$ & $\begin{array}{c}\mathbf{L}_{\exp } \\
(x \text {-fold })\end{array}$ & $\begin{array}{c}\mathbf{L}_{\text {calc }} \\
(x \text {-fold })\end{array}$ & $\boldsymbol{R}$ \\
\hline \multicolumn{7}{|l|}{ tRNA ${ }^{\text {Arg }}$ variants } \\
\hline $\operatorname{ArgWT}^{\mathrm{a}}$ & 0.68 & 550 & 1 & 1 & & \\
\hline $\operatorname{Arg} \cup 35^{a}$ & 0.0003 & 735 & 0.0005 & 2,670 & & \\
\hline $\operatorname{Arg} C 36^{a}$ & 0.014 & 520 & 0.022 & 45 & & \\
\hline $\operatorname{Arg} G 37^{a}$ & 0.30 & 605 & 0.4 & 2.5 & & \\
\hline ArgU35C36G37 & 0.0059 & 52 & 0.092 & 11 & $30 \times 10^{4}$ & $3.6 \times 10^{-5}$ \\
\hline "Hyb"a & 0.052 & 28,900 & 0.0015 & 670 & & \\
\hline "Hyb"C35 & 0.028 & 618 & 0.037 & 27 & & \\
\hline "Hyb"C35U36 & 0.45 & 1108 & 0.33 & 3 & & \\
\hline \multicolumn{7}{|l|}{ tRNA ${ }^{\text {Asp }}$ variants } \\
\hline$A s p W T^{\mathrm{a}}$ & 0.07 & 570 & 0.1 & 10 & & \\
\hline AspC35 ${ }^{\mathrm{a}}$ & 0.031 & 310 & 0.082 & 12 & & \\
\hline AspU36 ${ }^{\mathrm{a}}$ & 0.0006 & 1,415 & 0.0003 & 2,780 & & \\
\hline AspA37 ${ }^{a}$ & 0.0004 & 2,560 & 0.0001 & 7,230 & & \\
\hline AspU36A37 & 0.0004 & 324 & 0.001 & 990 & $20 \times 10^{6}$ & $4.9 \times 10^{-5}$ \\
\hline AspC35U36A37 & 0.19 & 118 & 1.30 & 0.8 & $241 \times 10^{6}$ & $1.24 \times 10^{-9}$ \\
\hline \multicolumn{7}{|l|}{ Architectural variants } \\
\hline Arg20/48 & 4.39 & 2,038 & 1.7 & 0.6 & & \\
\hline "Hyb"20/48 & 0.018 & 3,047 & 0.0047 & 210 & & \\
\hline
\end{tabular}

${ }^{a}$ Data from Sissler et al. (1996). Relative kinetic specificity constants are defined as $\left(k_{\text {cat }} / K_{m}\right)_{\text {relative }}=\left(k_{\text {cat }} / K_{m}\right)_{\text {mutant }} /$ $\left(k_{\text {cat }} / K_{m}\right)_{\text {wild-type. }} \mathbf{L}$ values are inverses of $\left(k_{\text {cat }} / K_{m}\right)_{\text {relative. }} \mathbf{R}$ values are defined as $\boldsymbol{R}=\mathbf{L}_{\text {exp }} / \mathbf{L}_{\text {calc. }}$ Values of $k_{\text {cat }}$ and $K_{m}$ were determined from Lineweaver and Burk plots and varied for replicate experiments at most $10 \%$. For all sets of kinetic analyses with tRNA variants, a control with ArgWT was run in parallel. All displayed data are mean values of at least two independent experiments and are normalized with regard to ArgWT. For nomenclature of variants, see footnote.

variabilities, a view supported by the crystallographic structure of the glutamine complex, representative of complexes with class I synthetases, in which nt 32 and 38 of tRNA ${ }^{\text {Gln }}$ are base paired and stacked over the anticodon stem (Rould et al., 1989, 1991).

In summary, the above results indicate that arginylation by yeast ArgRS can be governed by multiple effects combining information transduction from recognition sets in the anticodon loop to the catalytic site of the synthetase with differential participation of the tRNA bodies. There are at least four productive combinations of tRNA sequences that are aminoacylatable by ArgRS. In what follows, we examine in detail the contribution of identity elements and evaluate more precisely the role of the tRNA structures.

\section{Additive relationship between arginine recognition sets and tRNA frameworks}

An interesting relationship between ArgWT and AspWT appears when considering the tRNA as a structure built by two blocks, namely the framework (F) (as defined previously) and the N35-37 nucleotide triplet (T) containing the identity elements. Thus, ArgWT can be symbolized by ArgF/ArgT and AspWT by AspF/ AspT, and one tRNA appears derived from the other by two large mutations (Fig. 2). With the formalism $\mathbf{L}_{\text {calc }}(\mathrm{mm})=\boldsymbol{R} \Pi \mathbf{L}_{\text {exp }}(\mathrm{sm})$, where $\mathbf{L}$ 's are losses of catalytic efficiency of multiple $(\mathrm{mm})$ and single $(\mathrm{sm}) \mathrm{mu}$ - tants and $\boldsymbol{R}$ is a coupling factor accounting for deviations from additivity (Fersht, 1985; Pütz et al., 1993), one can calculate the impairment of arginylation for AspWT compared with ArgWT in two different ways. First, it can be calculated as the product of the loss of the triple tRNA ${ }^{\text {Arg }}$ mutant Arg U35C36G37 ( $\mathbf{L}=11)$ by that of AspWT with respect to this triple mutant $(\mathbf{L}=10 / 11=0.9)$.

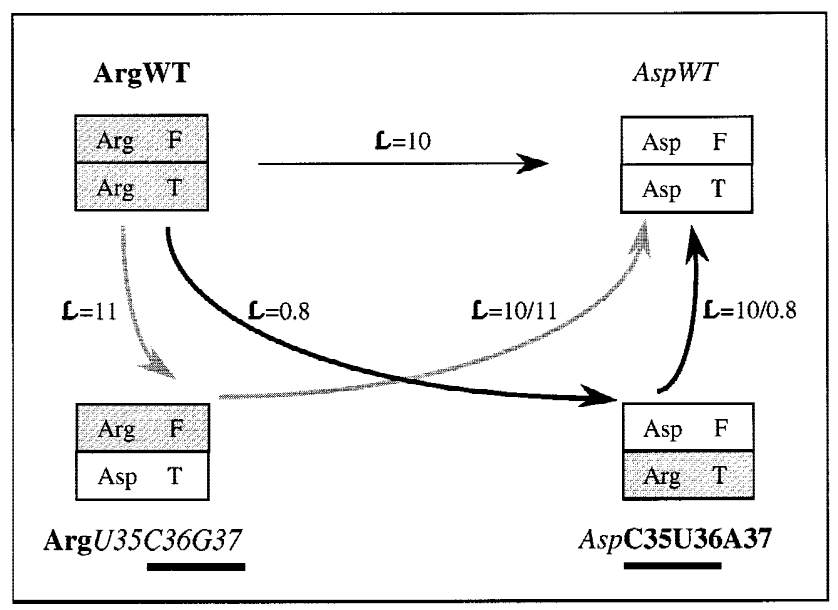

FIGURE 2. Additivity between structural blocks in tRNA substrates of yeast ArgRS. The two structural blocks constituting tRNA are symbolized by rectangles with $\mathrm{F}$ for frameworks and $\mathrm{T}$ for the N35-37 triplets. In the names of mutants with exchanged blocks, the recognition nucleotides are underlined. $\mathbf{L}$ values are taken from Table 1 and Figure 1. 
Second, it can be calculated using the alternate combination, with the loss of the "tRNA ${ }^{\text {Asp }}$ framework" mutant AspC35U36A37 ( $\mathbf{L}=0.8)$ multiplied by that of AspWT with respect to $\mathbf{A r g} U 35$ C36G37 ( $\mathbf{L}=10 \times$ $0.8=12.5)$. In both cases, the calculated $\mathbf{L}$ 's $\left[\mathbf{L}_{\text {calc }}=\right.$ $11 \times 0.9=9.9$ or $\mathbf{L}_{\text {calc }}=0.8 \times 12.5=10$ ] are equivalent to the experimental $\mathbf{L}=10$, which implies $\mathbf{R} \sim 1$ or additivity. In other words, arginine-accepting tRNAs are constituted by two structural blocks that act independently. Two combinations of blocks can lead to this additivity, namely the tRNA ${ }^{A r g}$ framework and the triplet from tRNA ${ }^{\text {Asp }}$, and conversely, the tRNA ${ }^{\text {Asp }}$ framework and the triplet from tRNA ${ }^{\text {Arg }}$.

\section{Anticooperative relationship between anticodon loop nucleotides}

To understand the contribution of individual anticodon loop nucleotides to arginylation, an analysis of partially transplanted tRNAs has been performed. Results are given in Table 1 and Figure 3. This analysis, again, is based on comparison of calculated versus experimental aminoacylation efficiencies of multiple mutants according to $\mathbf{L}_{\text {calc }}(\mathrm{mm})=\boldsymbol{R} \Pi \mathbf{L}_{\exp }(\mathrm{sm})$, with $\boldsymbol{R} \sim 1$ for additivity, $\boldsymbol{R}>1$ for cooperativity, and $\boldsymbol{R}<1$ for anticooperativity (Pütz et al., 1993).

Conversion of ArgWT to ArgU35C36G37 was done progressively (Fig. 3). Individual substitutions of C35 and U36 reduce arginylation, especially in ArgU35 $(\mathbf{L}=2670)$ and more moderately in Arg C36 ( $\mathbf{L}=45)$. These mutations were useful to define $\mathbf{C} 35$ as the major arginine recognition element and $\mathbf{U} 36$ as a minor element (Sissler et al., 1996). Replacement of $\mathbf{A} 37$ by G37 is without noticeable effect ( $\mathbf{L}=2.5$ in $\mathbf{A r g}$ G37). Simultaneous mutation of the three anticodon loop positions leads to efficient $\operatorname{Arg} U 35$ C36G37 $(\mathbf{L}=11)$. Comparison of its experimental efficiency with that calculated from single mutant data (Table 1) highlights an anticooperative relationship between the three nucleotides. Indeed, in the hypothesis of additivity, $\mathbf{L}_{\text {calc }}$ would be $2.5 \times 45 \times 2,670=3 \times 10^{5}$, a figure much higher than $\mathbf{L}_{\text {exp }}=11$. The coupling factor $\boldsymbol{R}$ of $3.6 \times 10^{-5}$ quantifies this strong effect. A simple interpretation would be that the anticooperativity is due to compensating effects brought by the mutations in the [C35U36] ${ }^{\text {Arg }}$. recognition set. A more subtle interpretation has to take into account that the mutations have replaced this set by the alternate [C36G37] ${ }^{\text {Asp }}$-set. Because footprinting shows proximity of identity nucleotides with ArgRS (Sissler et al., 1997), it is reasonable to assume that anticooperativity is due to replacement of the [C35U36]dependent pattern of contacts with ArgRS by the alternate [C36G37]-dependent pattern.

The same type of transplantation was done in the tRNA $^{\text {Asp }}$ context (Fig. 3). Four mutants were studied. Two of them, AspU36 and AspA37, are simple mutants. They have strongly impaired arginylation $(\mathbf{L}=$
2,780 and 7,230 , respectively), although the mutations have introduced in the aspartate framework residues naturally present in tRNA ${ }^{\mathrm{Arg}}$. The two other variants, AspU36A37 and AspC35U36A37, are double and triple mutants. In AspU36A37, the initial [C36G37] ${ }^{\text {Asp }}$. recognition set has been removed. As anticipated, this double variant is a poor substrate for ArgRS. However, impairment of its activity $(\mathbf{L}=990)$ is much lower than expected and anticooperativity occurs. Comparison of $\mathbf{L}_{\text {calc }}\left(2,780 \times 7,230=20 \times 10^{6}\right)$ with $\mathbf{L}_{\exp }(=990)$ quantifies the anticooperative relationship between U36 and $\mathbf{A} 37$ by a coupling factor $\boldsymbol{R}=4.9 \times 10^{-5}$. This strong anticooperativity can only result from compensatory effects brought by the mutations at the recognition positions because no positive signal has been introduced, except U36 considered as a minor identity element in the tRNA $A^{\text {Arg }}$ context. Replacement in tRNA ${ }^{\text {Asp }}$ of $U 35$ by $\mathbf{C} 35$ does not affect arginylation $(\mathbf{L}=12$ compared with $\mathbf{L}=10$ for AspWT). Because of the neutral effect of the C35 mutation, it could be anticipated that its introduction in the double mutant AspU36A37 would not improve its arginylation capacity. However, the resulting triple variant, AspC35U36A37, is a very efficient substrate $(\mathbf{L}=0.8)$. The calculated coupling factor $\boldsymbol{R}=1.24 \times 10^{-9}$ reveals here also strong anticooperative effects. Because AspC35U36A37 is bearing the complete [C35U36] ${ }^{\mathrm{Arg}}$-recognition set, it is reasonable to assume that anticooperativity is mediated by the creation of novel productive interactions with ArgRS.

In summary, transformation of wild-type tRNA ${ }^{\text {Arg }}$ or tRNA $^{\text {Asp }}$ transcripts into active chimera with exchanged arginine recognition sets results from strong anticooperative effects between nt 35, 36, and 37 that likely result from different adaptabilities of the tRNAs on ArgRS.

\section{Importance of nonrecognition residues on expression of arginine identity}

For a better perception of arginine identity, hybrid tRNAs combining the body of tRNA $A^{A r g}$ with wild-type or mutated versions of tRNA ${ }^{\text {Asp }}$-derived anticodon loops were studied. The results are presented in Table 1 and Figure 4. The reference molecule is ArgU35C36G37, which is a good arginine acceptor compared with ArgWT $(\mathbf{L}=11$, see above). Notice that the anticodon loop of this molecule is an Arg/Asp chimera with four residues from tRNA ${ }^{A r g}$ and three from tRNA ${ }^{\text {Asp }}$, namely dinucleotide C36G37, the alternate arginine recognition set $[\text { [C36G37] }]^{\text {Asp }}$, and $U 35$, which is a non-arginine nucleotide in the tRNA ${ }^{\text {Arg }}$ framework.

Activity of ArgU35C36G37 was first compared with that of three hybrid tRNAs (Fig. 4). The variant named "Hyb" contains the body of tRNA ${ }^{A r g}$ and the wild-type aspartate anticodon loop. The two other hybrids ("Hyb"C35 and "Hyb"C35U36) have, respectively, one and two residues from the [C35U36 $]^{\mathrm{Arg}}$-recognition 


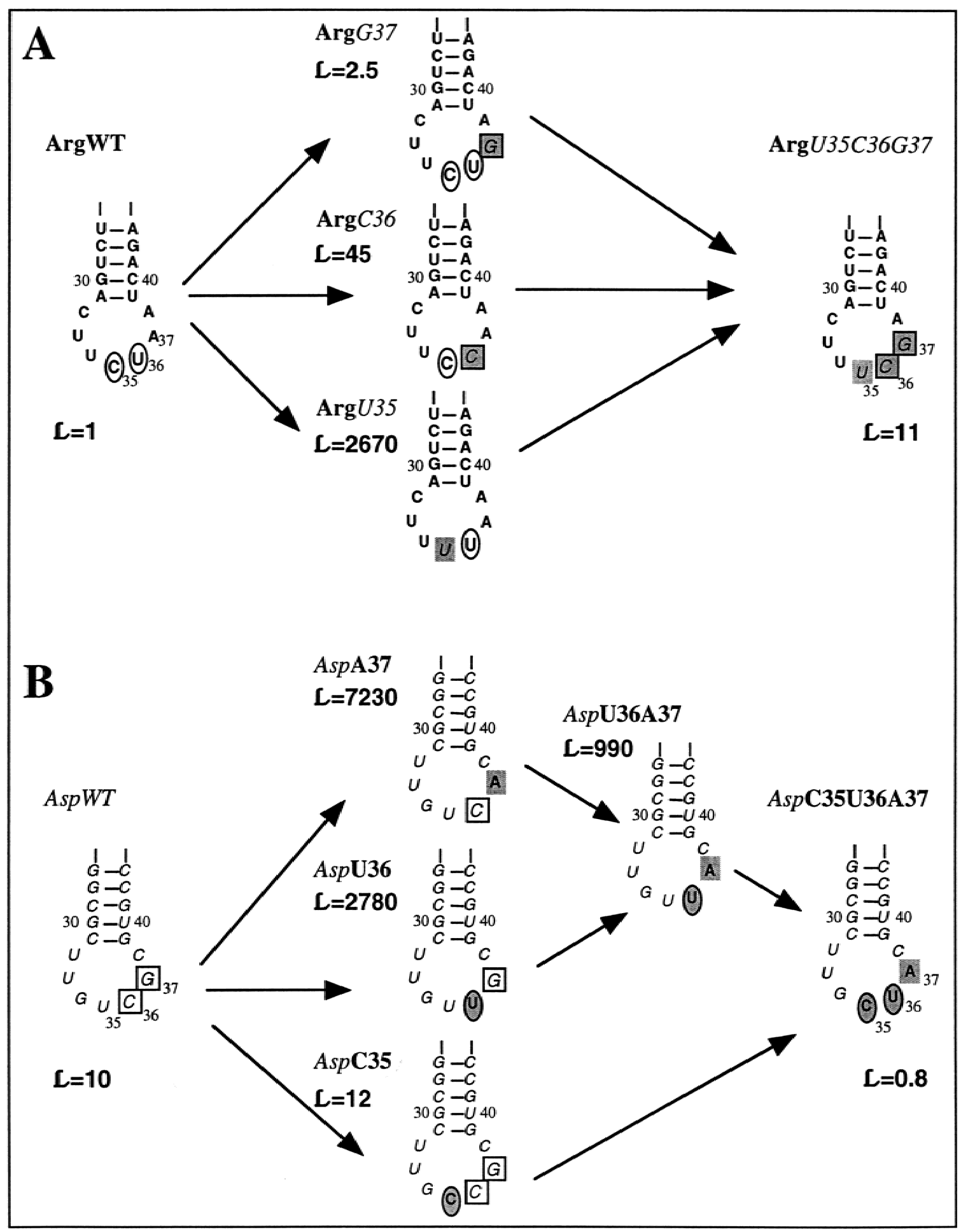

FIGURE 3. Anticooperativity between anticodon loop nucleotides in tRNA arginylation reactions catalyzed by yeast ArgRS. A: From tRNA ${ }^{\text {Arg }}$-recognition set [C35U36] ${ }^{\mathrm{Arg}}$ in ArgWT to tRNA ${ }^{\mathrm{Arg}}$-recognition set [C36G37] Asp $_{\text {in }}$ Arg U35C36G37 via single mutants ArgU35, Arg C36, and Arg G37. B: From tRNA ${ }^{A s p}$-recognition set [C36G37] $]^{\text {Asp }}$ in AspWT to tRNA ${ }^{\text {Asp }}$ recognition set [C35U36] Arg in AspC35U36A37 via single mutants AspC35, AspU36, and AspA37 and the double mutant AspU36A37. Graphic symbols are as in Figure 1.

set. As to "Hyb," its sole difference with Arg U35C36G37 concerns the three residues 32, 34, and 38, U33 being common to all tRNAs. Because "Hyb" contains the same arginine determinants as ArgU35C36G37 and because a neutral behavior of the changes in its anticodon loop (see Fig. 4) could be expected, a significant arginine activity for this variant was anticipated. On the contrary, "Hyb" shows a strong decrease in aminoacylation effi- 


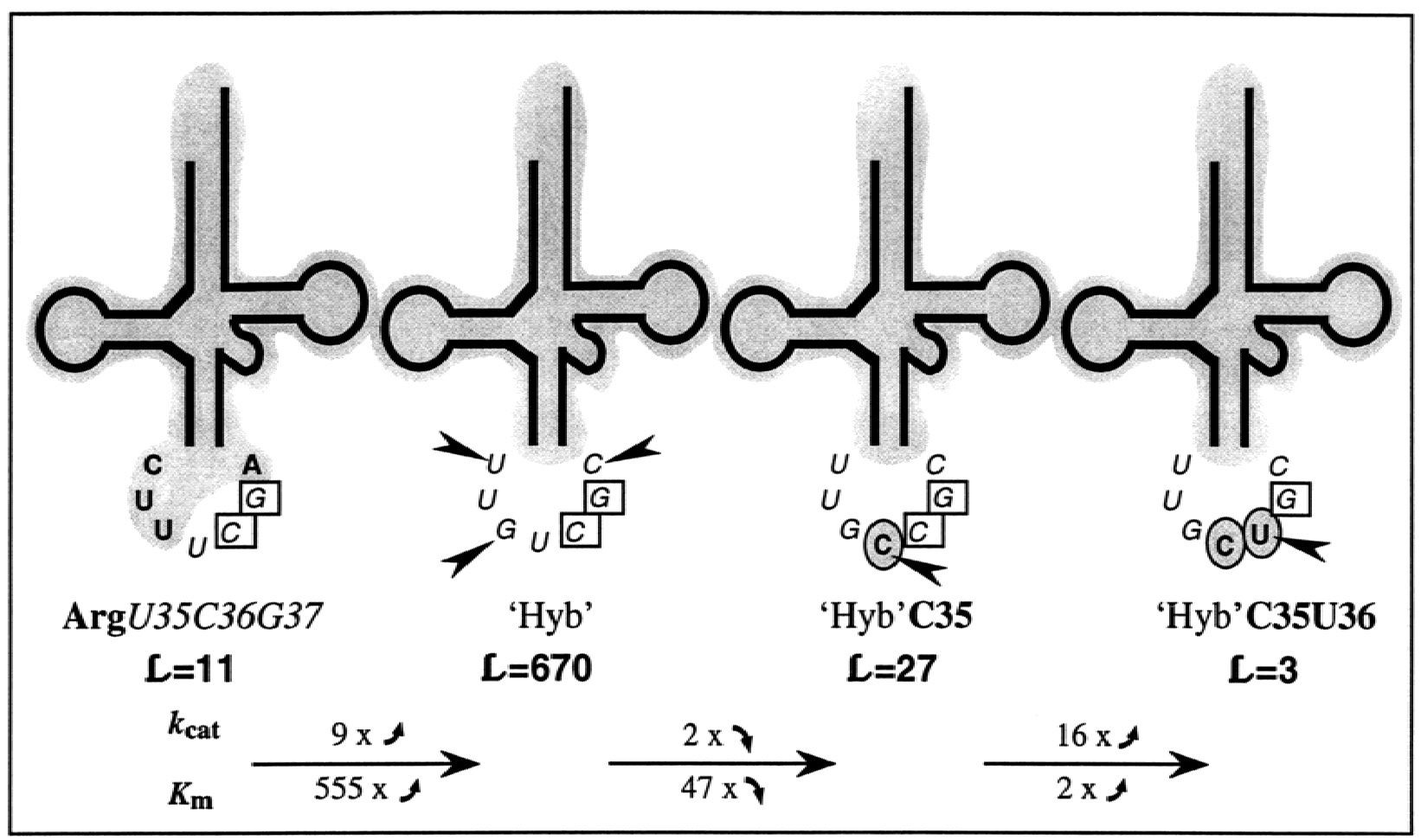

FIGURE 4. Effect of anticodon residues outside arginine recognition sets on aminoacylation by yeast ArgRS. The four tRNAs have the body of tRNA Arg (depicted as the backbone) with anticodon elements derived from either tRNA ${ }^{\text {Arg }}$ or tRNA ${ }^{\text {Asp }}$. Sequence elements derived from tRNA ${ }^{A r g}$ are on a grey background. Other typographic characters are as in Figure 1. The changes ( $x$-fold) of kinetic parameters between two variants (molecules at the left compared with their neighbors at the right) are indicated; arrows (up or down) symbolize that values of $k_{c a t}$ or $K_{m}$ are increased or decreased, respectively.

ciency $(\mathbf{L}=670)$ accounted for by a 13-fold decrease in $k_{c a t}$ and a 52 -fold increase in $K_{m}$. The correlated large loss in affinity, suggested by the increased $K_{m}$, reflects either the presence of strong negative elements and/or a drastic conformational change in the anticodon loop of this tRNA that hinders recognition by ArgRS. Whatever the exact reason for the poor activity of "Hyb," it is related to the tRNA sequence outside the U35C36G37 triplet that triggers activation of ArgRS. The same conclusion highlighting a major communication between anticodon loops and tRNA bodies arises when comparing activity of AspWT to that of "Hyb." Indeed, despite the fact that both tRNAs have the same anticodon loop, AspWT is 67-fold better arginylated than "Hyb" (compare $\mathbf{L}$ values given in Table 1 ).

The strong negative effect brought by the body of tRNA ${ }^{\text {Arg }}$ can be overcome in part by the single mutation $\mathrm{U} 35 \rightarrow \mathrm{C} 35$ ("Hyb"C35, Fig. 4). In this variant, arginylation efficiency is 25 -fold improved $(\mathbf{L}=27)$. This improvement, however, does not completely recover the activity $(\mathbf{L}=11)$ of the reference molecule. Insertion of C35 does not affect $k_{\text {cat }}$ significantly, but decreases $K_{m} \sim 50$-fold. It is worth recalling that, in the tRNA ${ }^{\text {Asp }}$ context, replacement of $U 35$ by $\mathbf{C} 35$ has no effect. Complete arginine activity is recovered by inserting U36 in "Hyb"C35 $(\mathbf{L}=3)$. In this fully active molecule, the real [C35U36] ${ }^{\text {Arg-recognition set reappears. }}$
Altogether, study of tRNA hybrids indicates a strong functional link between the body of tRNA ${ }^{\operatorname{Arg}}$ and the anticodon loop. It reveals also the crucial role of nonrecognition nucleotides in this loop for optimal expression of the [C36G37] ${ }^{\text {Asp }-r e c o g n i t i o n ~ s e t . ~}$

\section{Effect of the overall tRNA architecture on expression of arginine identity}

The transcripts of tRNA ${ }^{A r g} \mid I I$ and tRNA $A^{\text {Asp }}$ present structural differences in their $\mathrm{D}$-loops and variable regions, with the $\alpha / \beta$ domains of D-loops (at both sides of conserved G18G19) formed, respectively, by three/two and three/three nucleotides and variable regions formed by five and four nucleotides (see Fig. 1). These regions participate in 3D-folding of tRNA (Giegé et al., 1993) and account for conformational differences between tRNAs (e.g., Romby et al., 1985; Perret et al., 1992; Sissler et al., 1997) that were correlated with functional effects (e.g., Perret et al., 1992; Frugier et al., 1993, 1994). Along these lines, structural characteristics from tRNA $^{\text {Asp }}$ have been introduced into tRNA $A^{\text {Arg }}$ derivatives and their effect on arginylation has been measured (Table 1). An additional $U$ has been introduced between $\mathrm{G} 19$ and $\mathrm{C} 20$, and $\mathrm{U} 48$ has been removed from the variable region in both ArgWT (which becomes Arg20/48) and "Hyb" (which becomes "Hyb"20/48). 
Introduction of aspartate architectural features in ArgWT has rather moderate effects on arginylation, but closer analysis of the kinetic data reveals unpredicted features. Although an impairment of activity could be anticipated for Arg20/48, experiments in contrast indicate a gain in efficiency $(\mathbf{L}=0.6$ or $\mathbf{G} \sim 2$ ). For "Hyb"20/48, the effects are qualitatively similar, with a $\sim$ threefold increase in activity compared with "Hyb" ( $\mathbf{L}=210$ versus $\mathbf{L}=670$; Table 1 ). Interestingly, the better activities of the two architectural variants with reference to the control molecules (ArgWT and "Hyb") result from opposite variations of $k_{\text {cat }}$ and $K_{m}$ (Table 1 ). For Arg20/48, both $k_{c a t}$ and $K_{m}$ are increased $\sim$ sixand $\sim$ fourfold compared with ArgWT; for "Hyb"20/48, in contrast, they are decreased $\sim 3$ - and $\sim 10$-fold compared with "Hyb." This indicates subtle influences of the tRNA framework on expression of arginine identity.

The unexpected outcome of these experiments is the preference of ArgRS for architectural features from tRNA ${ }^{\text {Asp }}$, as evidenced by the better charging of Arg20/48 and "Hyb"20/48 than that of the control molecules with the arginine framework. Noticeable is the much decreased $K_{m}$, thus the increased affinity of "Hyb"20/48 for ArgRS in comparison with "Hyb," and, more surprising, the improvement of the catalytic turnover when ArgWT is mutated to Arg20/48.

\section{GENERAL CONCLUSIONS AND PERSPECTIVES}

Several conclusions highlighting mechanistic and evolutionary peculiarities of the arginine system emerge from the present study.

\section{Mechanistic aspects}

The fact that various tRNA constructs, differing either in their identity element content, their fine structure, and/or their sequence context, are all efficient substrates for ArgRS demonstrates that arginylation can proceed through various mechanistic routes. The two recognition sets [C35U36] ${ }^{\mathrm{Arg}}$ and [C36G37] ${ }^{\text {Asp }}$, which are in contact with ArgRS upon complex formation, are interchangeable within the two structural contexts of tRNA ${ }^{A r g} \mid I I$ and tRNA ${ }^{\text {Asp }}$ provided they are transplanted as a whole. The arginylation kinetics of the four efficient ArgRS substrates, i.e., the two wild-type tRNAs and the two chimera with exchanged recognition sets, reveal that the tRNAs are combinations of structural blocks. These blocks are the frameworks and the N35-37 triplets that behave independently. Closer analysis of the kinetic properties shows that the mechanisms by which arginine identity is expressed are not the same. Indeed, slower catalytic turnovers are compensated by better affinities of the tRNAs to ArgRS, an effect particularly pronounced for ArgU35C36G37. Conversely, faster turnovers are compensated by worse affinities.
From a structural point of view, this means that recognition of the nucleotide combinations accounting for arginine identity is accompanied by adequate adaptation between tRNAs and ArgRS. This adaptation is based on conformational changes of the nucleic acid, as demonstrated explicitly for ArgWT (Sissler et al., 1997).

Functional adaptation implies creation of appropriate contacts between recognition elements on RNA and protein. Because both kinetics and recognition elements differ in these tRNAs, their contacts with ArgRS likely are not the same. Thus, signals they switch on are transduced from their anticodon regions to the catalytic site of the synthetase, $-70 \AA$ apart, by different routes. Architectural features of the tRNA cores modulate this sequence-dependent process, which relies on the deformability of the nucleic acid. This is a common feature in protein-RNA recognition (Draper, 1995). Although the precise sequence of events leading to functional specificity is not yet unraveled, it is likely that these events include transconformation steps of the tRNA structure and thus are of the "induced fit" type. Indeed, individual arginine recognition elements have strongly anticooperative effects and nonrecognition elements are of importance for the expression of arginine identity. Such residues participate in the overall adaptation of the tRNA on the synthetases. Thus, subtle sequence relationships exist in arginine-accepting tRNAs. Here we have identified a number of them and we cannot exclude that other sequence combinations would also sustain efficient arginylation. Combinatorial methods will be required to explore these possibilities. Interestingly, the in vivo arginylation of an amber suppressor tRNA ${ }^{\text {Phe }}$ mutant lacking residue 26 was explained by a possible structural adjustment of the tRNA on the synthetase that allows simultaneous use of A20, the major arginine recognition element in Escherichia coli, and of an analogue of conserved C35, namely C34 from the CUA amber anticodon, which thus would contribute to arginine identity as well (McClain \& Foss, 1988). In line with the present work, this former study shows that $E$. coli ArgRS, like the yeast enzyme, can accommodate alternative recognition sets.

The great tolerance of ArgRS for a large variety of substrates, as described in the present report, has likely been amplified because of the greater flexibility of tRNA transcripts deprived of posttranscriptional modifications, compared with fully modified tRNAs (review by Agris, 1996). Thus, it is possible that the mechanistic routes governing arginylation in vivo are more restraint. Not only structural stabilization, but also protection against noncognate aaRS by posttranscriptional modification, as is typically the case for tRNA ${ }^{\text {Asp }}$ thanks to the presence of an antideterminant on position G37, could, in addition, influence acceptability of alternative identity nucleotide sets. Moreover, compensation of large $K_{m}$ effects by $k_{c a t}$ effects may not be overcome in vivo. 
However, the occurrence of five different tRNA ${ }^{\text {Arg }}$ isoacceptors in yeast, diverging largely at sequence and structural levels (see below), opens the possibility that ArgRS recognizes and charges each of the isoacceptors through alternate mechanistic routes. This, in turn, as well as the data obtained for the in vitro transcripts, opens the possibility of several combinations of amino acid identity sets at the level of the synthetase.

\section{Evolutionary aspects}

One can wonder why nature has established such intricate molecular recognition mechanisms for tRNA arginylation. In yeast, five isoaccepting tRNA ${ }^{A r g}$ species with large divergence in their sequences co-exist. Functional plasticity in the arginylation system opens the possibility that arginylation of each isoacceptor tRNA occurs through alternate mechanistic routes. This functional plasticity confers a biological advantage because arginylation efficiency may thus be less sensitive to mutations within tRNA or synthetase.

From these considerations, it follows that the phenomena and concepts discussed for the arginine system in yeast are of more general significance. tRNA aminoacylation systems are not completely idiosyncratic and are interconnected. The fact that this study was done on unmodified tRNA ${ }^{\text {Arg }}$ and tRNA ${ }^{\text {Asp }}$ transcripts has facilitated discovery of such interconnections. Even though major recognition nucleotides for tRNA identity can be discovered rather easily, it is shown here that other nucleotides in tRNA are not of completely neutral nature. Similar phenomena were recently reported in phenylalanylation systems (Kholod et al., 1997; Frugier et al., 1998). With E. coli PheRS, the recognition modes of cognate tRNA ${ }^{\text {Phe }}$ and phage T5 noncognate tRNA ${ }^{\text {Phe }}$ are dependent upon the $\mathrm{Mg}^{2+}$ level and thus are of different nature (Kholod et al., 1997). With yeast PheRS, tRNA charging is possible in different sequence contexts, provided the recognition set defined in cognate tRNA ${ }^{\text {Phe }}$ is present together with permissive elements in the acceptor stem of the engineered tRNAs (Frugier et al., 1998). Along the same lines, it was found in the $E$. coli alanine system that removal of the major recognition element can be compensated by a distal second mutation (Hou \& Schimmel, 1992). Further, synthetases can recognize structures far larger than canonical tRNA [e.g., tRNA-like structures in viruses (review by Florentz \& Giegé, 1995)] or obtained in vitro by artificial evolution (e.g., TinklePeterson et al., 1993). This potential has, in part, been erased by epigenetic phenomena (as in the yeast arginylation system by methylation of G37 in tRNA ${ }^{\text {Asp }}$ ) and by co-evolution of tRNAs for their interactions with other partners of the translational machinery. Knowledge of this potential of "functional plasticity" in tRNA aminoacylation systems encourages search of artificial tRNA substrates of synthetases that could become cellular inhibitors because they are unable to interact with the overall translational machinery.

Finally, the properties of yeast ArgRS reflect the evolutionary history of the arginylation system, in particular its relationship with the aspartate system and class II AspRS. First, we note the large sequence divergence between tRNA $A^{\text {Arg }}$ isoacceptors, as in yeast, that is conserved through evolution (see the sequence compilation by Sprinzl et al., 1998). Similarly, the importance of $\mathrm{C} 35$ for arginine identity was demonstrated in E. coli (Schulman \& Pelka, 1989; Tamura et al., 1992) and a functional link with structural characteristics of the D-loop was established (McClain \& Foss, 1988; Schulman \& Pelka, 1989; Tamura et al., 1992; McClain, 1993b). However, alternate recognition sets were not discovered to date in this organism, likely because no experiments were done on tRNA ${ }^{A r g}$ isoacceptors with G37, thus resembling tRNA ${ }^{\text {Asp }}$ in the anticodon loop. Following these lines, it is appealing to note the preference of ArgRS for structural characteristics present in tRNA ${ }^{\text {Asp }}$. Interestingly enough, tRNA ${ }^{A r g} I V$, a minor isoacceptor recently found in the yeast genome (Goffeau et al., 1996; Hani \& Feldmann, 1998), presents a high sequence homology with yeast tRNA ${ }^{\text {Asp }}(56 \%)$, much higher than that of the other arginine isocceptors ( $30 \%)$, with, in particular, G37 and the aspartate architectural characteristics in the D-loop and variable region ( $\alpha=\beta=3 ; \mathrm{v}=4$ ). This could mean that prototRNA $^{\text {Arg }}$ resembled tRNA ${ }^{\text {Asp }}$, or, expressed differently, that primordial class I ArgRS captured as substrate a tRNA, specific for the more ancient class II AspRS. If so, tRNA ${ }^{\text {Arg IV }}$ would be closest to such a primitive tRNA. In this context, is also remarkable to note an aspartate/ arginine relationship at the gene level, with tRNA ${ }^{\text {Arg III }}$ and tRNA ${ }^{\text {Asp }}$ originating from a same dimeric tRNA precursor in yeast (Schmidt et al., 1980). Although some of these correlations may be fortuitous, they provide a new conceptual frame to experimentally explore links between class I and class II tRNA aminoacylation systems.

\section{MATERIALS AND METHODS}

\section{Materials}

Nucleotides, deoxy-, and dideoxynucleotides were from Boehringer-Mannheim. Rotiphorese Gel 40 solution of acrylamide and $N, N^{\prime}$-methylene-bis-acrylamide (19:1) was from Carl Roth $\mathrm{GmbH}$ (Germany) and radioactive L- $\left[{ }^{3} \mathrm{H}\right]$ arginine ( $57 \mathrm{Ci} / \mathrm{mmol}$ ) was from Amersham. Yeast ArgRS was purified from an overproducing strain (Sissler et al., 1997) and had a specific activity of $4,000 \mathrm{U} / \mathrm{mg}$. T7 RNA polymerase was purified from an overproducing strain supplied by Dr. F.W. Studier (Brookhaven) according to Becker et al. (1996).

\section{Cloning and in vitro transcription}

tRNAs were obtained by in vitro transcription of synthetic genes. Genes encoding wild-type and variant species of yeast 
tRNA ${ }^{A r g}$ III and tRNA Asp, downstream from the T7 RNA polymerase promoter, were constructed and cloned according to

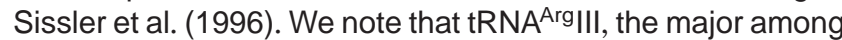
the five arginine isoacceptors, is represented by 11 of 19 tRNA $^{\text {Arg }}$ genes in the yeast genome (Goffeau et al., 1996; Hani \& Feldmann, 1998; G. Keith \& G. Dirheimer, in prep.). Sequences of tRNAs are according to Gangloff et al. (1971) and Keith and Dirheimer (1980). For tRNA ${ }^{\text {Asp }}$, it has been shown that replacement of U1-A72 by G1-C72, more favorable for transcription, is without detrimental consequence on its activity (Perret et al., 1990a). Plasmids containing genes for tRNAs were in vitro transcribed after linearization as described in Perret et al. (1990b). Transcripts were purified on $12 \%$ polyacrylamide/urea gels to single nucleotide resolution, electroeluted, and ethanol precipitated. Concentration of tRNA stock solutions were determined by absorbance measurements at $A_{260 \mathrm{~nm}}$.

\section{Aminoacylation reactions}

Aminoacylation reactions of transcripts derived from tRNA ${ }^{A r g}$ and tRNA ${ }^{\text {Asp }}$ have been performed as described in Sissler et al. (1996). Basically, from 0.2 to $4 \mu \mathrm{M}$ of transcripts were incubated in $50 \mathrm{mM}$ Hepes- $\mathrm{NaOH}, \mathrm{pH} 7.5,30 \mathrm{mM} \mathrm{KCl}$, $15 \mathrm{mM} \mathrm{MgCl}_{2}, 10 \mathrm{mM}$ ATP, $2.5 \mathrm{mM}$ glutathione, $50 \mu \mathrm{M}$ ${ }^{3} \mathrm{H}$-labeled arginine, and $0.3-900 \mathrm{nM}$ of yeast ArgRS. Notice that the amino acid concentration in aminoacylation assays is much higher than the $K_{m}$ of arginine, which is $1.5 \mu \mathrm{M}$ (Gangloff et al., 1976), so that kinetic artefacts are prevented.

\section{ACKNOWLEDGMENTS}

We are indebted to $G$. Eriani for discussions and for the overproducing strain of ArgRS, to G. Keith for useful comments on tRNA sequences, and to all our laboratory colleagues for valuable advice. We thank $A$. Hoeft for the synthesis of oligonucleotides. This work was partly supported by grants from the Centre National de la Recherche Scientifique (CNRS), the Ministère de l'Enseignement Supérieur et de la Recherche (MESR), and Université Louis Pasteur (Strasbourg). M.S. was supported by MESR and Association pour la Recherche contre le Cancer (ARC) grants.

Received January 5, 1998; returned for revision February 2, 1998; revised manuscript received March 5, 1998

\section{REFERENCES}

Agris PF. 1996. The importance of being modified: Roles of modified nucleosides and $\mathrm{Mg}^{2+}$ in RNA structure and function. Prog $\mathrm{Nu}$ cleic Acid Res Mol Biol 53:79-129.

Becker HD, Giegé R, Kern D. 1996. Identity of prokaryotic and eukaryotic tRNA ${ }^{A s p}$ for aminoacylation by aspartyl-tRNA synthetase from Thermus thermophilus. Biochemistry 35:7447-7458.

Dirheimer G, Keith G, Dumas P, Westhof E. 1995. Primary, secondary and tertiary structures of tRNAs. In: Söll D, RajBhandary UL, eds. tRNA: Structure, biosynthesis, and function. Washington, DC: Am Soc Microbiol Press. pp 93-126.

Draper DE. 1995. Protein-RNA recognition. Annu Rev Biochem 64:593-620.

Ebel JP, Giegé R, Bonnet J, Kern D, Befort N, Bollack C, Fasiolo F, Gangloff J, Dirheimer G. 1973. Factors determining the specificity of the tRNA aminoacylation reaction. Biochimie 55:547-557.
Fersht A. 1985. Enzyme, structure and mechanism. New York: Freeman.

Florentz C, Giegé R. 1995. tRNA-like structures in viral RNAs. In: Söll D, RajBhandary UL, eds. tRNA: Structure, biosynthesis, and function. Washington, DC: Am Soc Microbiol Press. pp 141-163.

Frugier M, Florentz C, Schimmel P, Giegé R. 1993. Triple aminoacylation specificity of a chimerized transfer RNA. Biochemistry 32:14053-14061.

Frugier M, Helm M, Felden B, Giegé R, Florentz C. 1998. Sequences outside recognition sets are not neutral for tRNA aminoacylation: Evidence for non-permissive combinations of nucleotides in the acceptor stem of yeast tRNA ${ }^{\text {Phe }}$. J Biol Chem. Forthcoming.

Frugier M, Söll D, Giegé R, Florentz C. 1994. Identity switches between tRNAs aminoacylated by class I glutaminyl- and class II aspartyl-tRNA synthetases. Biochemistry 33:9912-9921.

Gangloff J, Ebel JP, Dirheimer G. 1973. Isolation of a complex between yeast arginyl-tRNA synthetase and yeast tRNA ${ }^{A s p}$, and mischarging of tRNA ${ }^{A s p}$ with arginine. Internat Res Commun System 12:8.

Gangloff J, Keith G, Ebel JP, Dirheimer G. 1971. Structure of aspartate tRNA from brewer's yeast. Nature New Biol 230:125-127.

Gangloff J, Schutz A, Dirheimer G. 1976. Arginyl-tRNA synthetase from baker's yeast. Purification and some properties. Eur J Biochem 65:177-182.

Giegé R, Puglisi JD, Florentz C. 1993. tRNA structure and aminoacylation efficiency. Prog Nucleic Acid Res Mol Biol 45:129-206.

Goffeau A, Barrell BG, Bussey H, Davis RW, Dujon B, Feldmann H, Galibert G, Hoheisel JD, Jacq C, Johnston M, Louis EJ, Mewes HW, Murakami Y, Philippsen P, Tettelin H, Oliver SG. 1996. Life with 6000 genes. Science 274:546-567.

Hani J, Feldmann H. 1998. tRNA genes and retroelements in the yeast genome. Nucleic Acids Res 26:689-696.

Hou YM, Schimmel P. 1992. Functional compensation of a recognitiondefective transfer RNA by distal base pair substitution. Biochemistry 31:10310-10314.

Keith G, Dirheimer G. 1980. Reinvestigation of the primary structure of brewer's yeast tRNA ${ }^{\text {Arg } I I I . ~ B i o c h e m ~ B i o p h y s ~ R e s ~ C o m m u n ~}$ 92:116-119.

Kholod NS, Pan'khova NV, Mayorov SG, Krutilina AI, Shlyapnikov MG, Kisselev LL, Ksenzenko VN. 1997. Transfer RNAPhe isoacceptors possess non-identical set of identity elements at high and low $\mathrm{Mg}^{2+}$ concentration. FEBS Lett 411:123-127.

McClain WH. 1993a. Transfer RNA identity. FASEB J 7:72-78.

McClain WH. 1993b. Rules that govern tRNA identity in protein synthesis. J Mol Biol 234:257-280.

McClain WH, Foss K. 1988. Changing the acceptor identity of a transfer RNA by altering nucleotides in a "variable pocket." Science 241:1804-1807.

Perret V, Florentz C, Puglisi JD, Giegé R. 1992. Effect of conformational features on the aminoacylation of tRNAs and consequences on the permutation of tRNA specificities. J Mol Biol 226:323-333.

Perret V, Garcia A, Grosjean H, Ebel JP, Florentz C, Giegé R. 1990a. Relaxation of transfer RNA specificity by removal of modified nucleotides. Nature 344:787-789.

Perret V, Garcia A, Puglisi J, Grosjean H, Ebel JP, Florentz C, Giegé R. 1990b. Conformation in solution of yeast tRNA ${ }^{\text {Asp }}$ transcripts deprived of modified nucleotides. Biochimie 72:735-744.

Pütz J, Florentz C, Benseler F, Giegé R. 1994. A single methyl group prevents the mischarging of a tRNA. Nature Struct Biol 1:580582.

Pütz J, Puglisi JD, Florentz C, Giegé R. 1993. Additive, cooperative and anti-cooperative effects between identity nucleotides of a tRNA. EMBO J 12:2949-2957.

Romby P, Moras D, Bergdoll M, Dumas P, Vlassov VV, Westhof E, Ebel JP, Giegé R. 1985. Yeast tRNA ${ }^{\text {Asp }}$ tertiary structure in solution and areas of interaction of the tRNA with aspartyl-tRNA synthetase. A comparative study of the yeast phenylalanine system by phosphate alkylation experiments with ethylnitrosourea. $J \mathrm{Mol}$ Biol 184:455-471.

Rould MA, Perona JJ, Söll D, Steitz TA. 1989. Structure of E. coli glutaminyl-tRNA synthetase complexed with tRNA ${ }^{\text {Gln }}$ and ATP at 2.8 A resolution. Science 246:1135-1142.

Rould MA, Perona JJ, Steitz TA. 1991. Structural basis of anticodon loop recognition by glutaminyl-tRNA synthetase. Nature 352:213218. 
Saks ME, Sampson JR, Abelson JN. 1994. The transfer RNA identity problem: A search for rules. Science 263:191-197.

Schulman LH, Pelka H. 1989. The anticodon contains a major element of the identity of arginine transfer RNAs. Science 246:15951597.

Sissler M, Eriani G, Martin F, Giegé R, Florentz C. 1997. Mirrorimage alternate interaction patterns of a same tRNA with either class I arginyl-tRNA synthetase or class II aspartyl-tRNA synthetase. Nucleic Acids Res 25:4899-4906.

Sissler M, Giegé R, Florentz C. 1996. Arginine aminoacylation identity is context-dependent and ensured by alternate recognition sets in the anticodon loop of accepting tRNA transcripts. EMBO J 15:5069-5076.
Schmidt O, Mao Ji, Ogden R, Beckmann J, Sakano H, Abelson J, Söll D. 1980. Dimeric tRNA precursors in yeast. Nature 287:750752.

Sprinzl M, Horn C, Brown M, loudovitch, Steinberg S. 1998. Compilation of tRNA sequences and sequences of tRNA genes. Nucleic Acids Res 26:148-153.

Tamura K, Himeno H, Asahara H, Hasegawa T, Shimizu M. 1992. In vitro study of E. coli tRNA ${ }^{\text {Arg }}$ identity. Nucleic Acids Res 20:23352339.

Tinkle-Peterson E, Blank J, Sprinzl M, Uhlenbeck O. 1993. Selection of active $E$. coli tRNA ${ }^{\text {Phe }}$ variants from a randomized library using two proteins. EMBO J 12:2959-1967. 

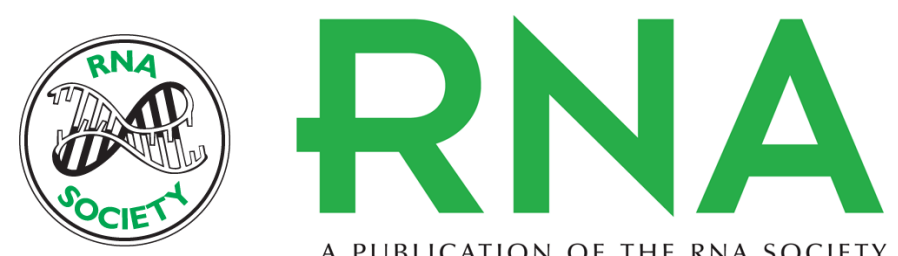

A PUBLICATION OF THE RNA SOCIETY

\section{The RNA sequence context defines the mechanistic routes by which yeast arginyl-tRNA synthetase charges tRNA.}

M Sissler, R Giegé and C Florentz

RNA 1998 4: 647-657

\section{License}

Email Alerting Receive free email alerts when new articles cite this article - sign up in the box at the Service top right corner of the article or click here.

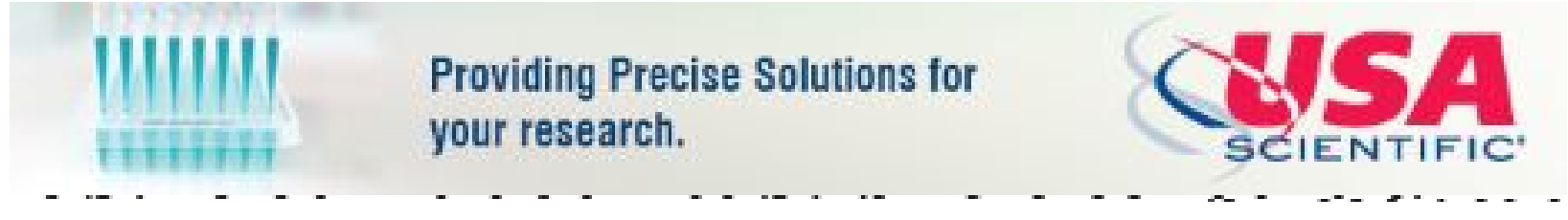

